\title{
A sonolência está associada a sintomas de ansiedade em escolares de 9 a 11 anos?
}

\author{
Is sleepiness associated with anxiety symptoms in schoolchildren aged 9 to 11 years? \\ ¿La somnolencia se associa con síntomas de ansiedad en escolares de 9 y 11 años?
}

Flávio Henrique de Santana

ORCID: https://orcid.org/0000-0001-7851-8975

Universidade Federal de Pernambuco, Brasil

E-mail: flvshenrique@gmail.com

Lívia Maria de Lima Leôncio

ORCID: https://orcid.org/0000-0001-9725-7061

Faculdade Venda Nova Imigrante, Brasil

E-mail: livia.lm1119@gmail.com

Clécia Gabriela Bezerra

ORCID: https://orcid.org/0000-0002-3747-7713

Universidade Federal de Pernambuco, Brasil

E-mail: clecia.bezerra@ufpe.br

Gilberto Ramos Vieira

ORCID: https://orcid.org/0000-0002-5155-3532

Universidade Federal de Pernambuco, Brasil

E-mail: gilberto_r.2010@hotmail.com

Letycia dos Santos Neves

ORCID: https://orcid.org/0000-0003-2286-7986

Universidade Federal de Pernambuco, Brasil

E-mail: letycia.sneves@gmail.com

Marina Souza Barbosa de Mattos

ORCID: https://orcid.org/0000-0002-4445-0754

Universidade Estadual da Paraíba, Brasil

E-mail: marina09mattos@gmail.com

Nataly Ferreira dos Santos

ORCID: https://orcid.org/0000-0001-8676-8114

Faveni, Brasil

E-mail: nataly.psi@outlook.com

Mírian Celly Medeiros Miranda David

ORCID: https://orcid.org/0000-0002-7152-8852

Universidade Federal de Pernambuco, Brasil

E-mail: miriancelly@hotmail.com

Rhowena Jane Barbosa de Matos

ORCID: https://orcid.org/0000-0001-5914-7304

Universidade Federal do Recôncavo da Bahia, Brasil

E-mail: rhowena.matos@ufrb.edu.br

\begin{abstract}
Resumo
Problemas relacionados ao sono na infância podem predizer distúrbios emocionais e comportamentais posteriores, como ansiedade. Sendo assim, objetivou-se investigar a relação da sonolência sobre os sintomas de ansiedade ao longo do semestre em escolares de 9 a 11 anos de idade. Foram avaliadas crianças matriculadas em uma escola pública, localizada no município de Vitória de Santo Antão - Pernambuco, de ambos os sexos, sem distúrbios do sono, deficiência física e distúrbios cognitivos $(\mathrm{n}=88)$. Para a análise do humor, foi utilizado o Inventário de Ansiedade Traço- Estado. A avaliação subjetiva do sono foi realizada por meio da Escala de Sonolência de Epworth e o Questionário de Matutinidade e Vespertinidade de Horne \& Ostberg (QMV-HO). Os questionários foram aplicados no período da manhã, no início (P1) e no final (P2) do semestre letivo, com exceção do QMV-HO. A correlação entre sonolência e estado de ansiedade no período $1(\mathrm{r}=0,16 ; \mathrm{p}=0,21)$, foi insuficiente, bem como no período 2 ( $\mathrm{r}=0,34$; $\mathrm{p}=0,01$ ). Houve baixa correlação entre sonolência e os traços de ansiedade no período 1, contanto a correlação foi moderada no período 2 ( $\mathrm{r}=0,48 ; \mathrm{p}<0,001)$. Nos aspectos positivos e negativos da ansiedade, a correlação da sonolência e o traço e estado de ansiedade apresentaram-se com correlação de baixa a moderada. Os dados revelam que os alunos avaliados apresentam anormalidade na sonolência diurna no final do semestre letivo e tais alterações podem piorar o traço de ansiedade nas crianças avaliadas.
\end{abstract}

Palavras-chave: Sono; Emoções; Ansiedade; Criança. 


\begin{abstract}
Childhood sleep-related problems can predict later emotional and behavioral disturbances, such as anxiety. Thus, this study aimed to investigate the relationship between sleepiness and anxiety symptoms throughout the semester in schoolchildren aged 9 to 11 years old. Children enrolled in a public school, located in Vitória de Santo Antão, Pernambuco, Brazil, of both genders, without sleep disorders, physical disabilities or cognitive disorders $(n=88)$ were evaluated. The State-Trait Anxiety Inventory was used to analyze mood. Subjective sleep assessment was performed using the Epworth Sleepiness Scale and the Horne \& Ostberg Morningness and Eveningness Questionnaire (MEQ-HO). The questionnaires were applied in the morning at the beginning (P1) and at the end (P2) of the school semester, with the exception of the MEQ-HO. The correlation between sleepiness and state of anxiety in period 1 $(\mathrm{r}=0.16 ; \mathrm{p}=0.21)$ was insufficient, as well as in period $2(\mathrm{r}=0.34 ; \mathrm{p}=0.01)$. There was a low correlation between sleepiness and anxiety traits in period 1 , while the correlation was moderate in period $2(r=0.48 ; p<0.001)$. The correlation between sleepiness and the trait and state of anxiety presented a low to moderate correlation in the positive and negative aspects of anxiety. The data reveal that the evaluated students present abnormal daytime sleepiness at the end of the school semester and such alterations can worsen the anxiety trait in the evaluated children. Keywords: Sleep; Emotions; Anxiety; Child.
\end{abstract}

\begin{abstract}
Resumen
Los problemas relacionados con el sueño en la infancia pueden predecir trastornos emocionales y conductuales posteriores, como la ansiedad. Así, el objetivo de este estudio fue investigar la relación entre la somnolencia y los síntomas de ansiedad durante el periodo académico en escolares de 9 a 11 años de edad. Fueron evaluados niños matriculados en una escuela pública, localizada en el municipio de Vitória de Santo Antão - Pernambuco, de ambos sexos, sin alteraciones del sueño, discapacidad física o deterioro cognitivo $(n=88)$. Para evaluación del estado de ánimo, fue utilizado el Inventario de Ansiedad Rasgo-Estado. La evaluación subjetiva del sueño fue realizada a través de la Escala de Somnolencia de Epworth y el Cuestionario matutino y vespertino de Horne \& Ostberg (QMV-HO). Los cuestionarios fueron aplicados en el periodo de la mañana, en el inicio (P1) y el final (P2) del periodo escolar, con excepción del QMV-HO. La correlación entre somnolencia y ansiedad estado no período 1 ( $\mathrm{r}=0,16 ; \mathrm{p}=0,21)$, fue insuficiente, así como en el periodo $2(\mathrm{r}=0,34 ; \mathrm{p}=0,01)$. Hubo una baja correlación entre la somnolencia y ansiedad rasgo en el periodo 1, mientras que la correlación fue moderada en el periodo 2 ( $r=0,48 ; \mathrm{p}<0,001)$. En los aspectos positivos y negativos de la ansiedad, la correlación de la somnolencia y ansiedad estado-rasgo mostraron una correlación de baja a moderada. Los resultados revelan que los alumnos evaluados presentan una alteración en la somnolencia diurna en el final del periodo escolar, y estas pueden agudizar la ansiedad rasgo de los escolares.
\end{abstract}

Palabras clave: Sueño; Emociones; Ansiedad; Niño.

\title{
1. Introdução
}

O ritmo biológico é definido como qualquer expressão fisiológica e comportamental presente em um determinado período regular, como por exemplo, o ciclo sono-vigília, ciclo menstrual, secreção hormonal e até mesmo a regularidade alimentar do indivíduo (Reyes et al., 2018). Alguns fatores estão diretamente relacionados à regulação do ritmo biológico, sejam eles endógenos (como os hormônios), e/ou os exógenos (como o sol que é um sincronizador geofísico). Os osciladores que exercem essas influências rítmicas sobre o organismo vivo podem ser denominados também de "zeitgeber", palavra em alemão que significa “doador de tempo". Essa interação, entre fatores endógenos e exógenos sob o ritmo biológico, irá depender de alguns osciladores como alimentação, exposição à iluminação, prática de exercício físico, etc. (Reyes et al., 2018).

O ser humano está, ao longo da sua vida, gerando ritmos biológicos. Estes podem durar um dia, conhecido como ritmo circadiano; podem exceder o período de $24 \mathrm{~h}$, denominado de ritmo infradiano; ou ter um período mais curto e frequência maior que o ritmo circadiano, o ritmo ultradiano (Bueno \& Wey, 2018).

O ritmo circadiano caracteriza-se por ter um ciclo que atua simultaneamente com fatores exógenos e completa-se dentro de um período médio de 24 horas (Almodes \& Araújo, 2003). Existe variação de expressão do ritmo de acordo com a idade do indivíduo, por exemplo, na infância a ritmicidade é mais sistematizada; já na velhice, esta é mais fragmentada e enfraquecida (Bueno \& Wey, 2018). Cada indivíduo tem predisposição natural de sentir picos de energia ou cansaço de acordo com a hora do dia. A regularidade dos estímulos externos pode favorecer as funções, também regulares, do organismo durante 
o período de mais ou menos vinte e quatro horas, no chamado ciclo circadiano, a exemplo do ciclo sono-vigília (MennaBarreto \& Marques, 2002).

O ciclo sono-vigília é regulado pelo ciclo claro e escuro ambiental (fase do dia e noite) (Pavlova, 2017). Nesse processo há a ação de uma variabilidade de sinalizadores neurais e periféricos, como o cortisol e a melatonina. Ao despertar, há uma elevação dos níveis de cortisol, conhecido como hormônio de atividade, muitas vezes relacionados ao estresse. Este é produzido e liberado pelas células da camada fasciculada das glândulas suprarrenais. O cortisol favorece o aumento da atividade celular, preparando o organismo para o estado de vigília (Kim \& Duffy, 2018). Com a redução da luminosidade e chegada da noite, os níveis de melatonina (hormônio precursor do sono) produzidos na glândula pineal do epitálamo, tendem a aumentar, levando o indivíduo a sentir-se mais sonolento (Kim \& Duffy, 2018).

Essa atividade recorrente e que cada indivíduo apresenta, pela cronobiologia, denomina-se cronotipo. Conceito relacionado com a disposição funcional que os indivíduos possuem para desenvolver suas atividades com maior eficiência e sentem-se melhor em sua realização (Martins \& Gomes, 2010). Os indivíduos podem ser classificados de acordo com o cronotipo em: matutinos, que dormem e acordam mais cedo e sentem-se mais dispostos pela manhã; vespertinos, que tendem a dormir e acordar mais tarde, com melhor desempenho em atividades nos turnos mais tardios; e os indiferentes ou intermediários, que não apresentam preferência de horário para melhor desempenho. Apesar disso, os seres vivos passam por um ciclo diário caracterizado pela alternância entre o período que estão acordados e o período em que estão dormindo. Isto pode variar de acordo com sua idade e rotina, sendo conhecido como ciclo sono-vigília (Martins \& Gomes, 2010; Campos et al., 2011).

O sono é reconhecido como um comportamento multidirecional, ou seja, influencia várias funções do organismo, como os aspectos cognitivos e as emoções. Além disso, essas funções podem ser beneficiadas durante o sono (Gustafsson et al., 2016). O funcionamento adequado do organismo está relacionado à boa qualidade do sono. Portanto, quando há insuficiência na quantidade de horas dormidas, o indivíduo torna-se mais suscetível a desenvolver um distúrbio do sono, ou esse perfil pode refletir o distúrbio instalado (Xião et al., 2020).

As recomendações para a duração do sono variam de acordo com a faixa etária do indivíduo, sendo recomendado 9 a 10 horas de sono por dia para crianças no período escolar (Heins et al., 2016). A prevalência de hábitos de sono problemáticos em crianças escolares varia entre 10 e 30\% (Owens \& Mindell, 2011; Silva et al., 2013). A insuficiência do sono dificulta a manutenção do estado de vigília adequado ao longo do dia, principalmente em crianças e adolescentes (Fallone et al., 2002; Moreira \& Pradella-Hallinan, 2017).

A desregulação do sono pode desencadear problemas emocionais nas crianças, tendo forte associação com o aparecimento de sintomas de ansiedade (Iwadare et al., 2015). Além disso, quando essas desordens emocionais não são tratadas ainda na infância, podem prejudicar o indivíduo até a vida adulta (Mcmakin \& Alfano, 2015).

Emoções são um conjunto de respostas neurais que surgem quando o cérebro é estimulado por fatores externos e internos (Gondim et al., 2015). A regulação emocional contribui para o desenvolvimento saudável das crianças; melhora a capacidade de relacionar-se no cotidiano; otimiza as habilidades escolares; e potencializa o desenvolvimento cognitivo (Callear et al., 2018). Alterações nos processos emocionais podem provocar consequências negativas ao indivíduo, pois podem afetar funções cognitivas (Gondim et al., 2015).

A falta de sono pode condicionar o tipo de reação emocional a uma situação geradora de stress (Gustafsson et al., 2016). Essas situações podem interferir diretamente nas relações pessoais e podem causar dificuldades na aprendizagem, sendo prejudicial para o desempenho no âmbito escolar (Halal \& Nunes, 2014). Além disso, pode levar ao desenvolvimento de transtornos do humor, como o transtorno de ansiedade (Kirwan et al., 2017). 
A ansiedade pode ser definida como um sentimento de desconforto, muitas vezes derivado da antecipação do perigo ou risco. Na idade escolar é comum as crianças relatarem medo de variados tipos de condições que em sua concepção possa vir a levá-las a uma situação de perigo, como medo de escuro, altura, animais perigosos etc. O estresse das atividades escolares e esses fatores citados podem vir a desencadear sintomas de ansiedade nas crianças, prejudicando negativamente suas vidas (Oliveira, 2017). Tendo em vista que a ansiedade pode afetar o indivíduo durante qualquer fase da vida, sua identificação ainda durante a infância torna-se valiosa para que haja um tratamento adequado que possa melhorar sua qualidade de vida.

Um sono regulado e adequado está associado a uma boa qualidade de vida, sendo importante para a manutenção do bem-estar físico e mental (Moro et al., 2020). A má qualidade de sono está relacionada a emoções negativas, como a raiva e o medo, provocando maior dificuldade de controlá-las e, consequentemente, interferindo negativamente no seu bem-estar físico, social e mental (Palmer \& Alfano, 2017).

Estudos recentes são importantes para conhecer como os problemas de sono influenciam as emoções, tendo em vista que os distúrbios do sono e problemas emocionais estão mais frequentes. Entretanto, há poucos estudos recentes relacionando a influência da qualidade do sono sobre as emoções em escolares. Portanto, hipotetiza-se que a má qualidade do sono esteja relacionada à desregulação do sono, possibilitando o aparecimento de transtornos de humor, como a ansiedade, e interferindo na vida social e escolar das crianças. Diante disso, objetivou-se investigar a relação da sonolência sobre os sintomas de ansiedade ao longo do semestre escolar em crianças de 9 a 11 anos.

\section{Metodologia}

\subsection{Desenho do estudo, população e amostra}

Trata-se de um estudo analítico, longitudinal, parte de outro estudo maior intitulado "Análise dos níveis de ingestão de água e a relação entre aspectos cognitivos, ritmo de sono-vigília e aptidão física de alunos com idade entre 9 e 11 anos, realizado em uma escola pública, localizada na área urbana da cidade de Vitória de Santo Antão - PE”. Este estudo foi conduzido entre agosto e novembro de 2019 , com crianças pertencentes do $3^{\circ}$ ao $5^{\circ}$ ano da escola. Incluiu-se crianças entre 9 e 11 anos e 11 meses, de ambos os sexos, cujas tiveram o consentimento dos pais para participarem. Excluiu-se as crianças com: (a) diagnósticos prévios de distúrbios no sono, deficiências físicas, distúrbios cognitivos, ansiedade ou depressão; (b) quaisquer situações clínicas e funcionais que impossibilitassem sua participação no estudo; (c) alunos que desistiram de participar da pesquisa, ao longo do semestre.

A população de alunos matriculados na escola é de 1291 alunos, sendo 334 estudantes matriculados do $3^{\mathrm{a}}$ ao $5^{\mathrm{a}}$ ano do turno da manhã, onde desse total, apenas 88 alunos preencheram os critérios de inclusão e tiveram autorização dos pais para participar da pesquisa. Todos os procedimentos desta pesquisa foram de acordo com as Diretrizes e Normas Regulamentadoras de Pesquisa Envolvendo Seres Humanos (196/96) editadas pela Comissão Nacional de Saúde, que atenderam as recomendações da Resolução 466/12. A pesquisa foi aprovada pelo comitê de ética de pesquisa em seres humanos da Universidade Federal de Pernambuco sob o protocolo: CAAE 26612919.9.0000.5208.

\subsection{Operacionalização das coletas}

Inicialmente, os pais dos alunos do $3^{\mathrm{a}}$ ao $5^{\mathrm{a}}$ ano, foram convocados para uma reunião coletiva, cuja finalidade foi esclarecer os objetivos e abordagem da pesquisa. Só então foi entregue e solicitada a assinatura do Termo de consentimento livre e esclarecido (TCLE) e do Termo de assentimento livre e esclarecido (TALE) aos pais dos alunos. Após a concordância e assinatura dos termos, foi feita a coleta dos dados gerais dos participantes, por meio da aplicação de questionário sociodemográfico; 
Os testes aplicados nesta pesquisa foram conduzidos por uma equipe devidamente treinada, cuja foi composta por graduandos da área da saúde, técnico de enfermagem e psicóloga, sendo acompanhados diariamente por docentes com uma vasta experiência em pesquisas na área. Dessa maneira, a aplicação dos testes foi dividida em dois períodos. O primeiro foi realizado em dois dias no início do semestre (em agosto); e o segundo, em dois dias do final do semestre (em novembro):

Início do semestre (P1): Foram aplicados o Inventário de Ansiedade Traço-Estado (IDATE), a Escala de Sonolência de Epworth (ESE) e o Questionário de Matutinidade e Vespertinidade de Horne \& Ostberg (QMV-HO).

Final do semestre (P2): Repetiu-se a aplicação do Inventário de Ansiedade Traço-Estado (IDATE) e a Escala de Sonolência de Epworth (ESE).

\subsection{Descrição dos instrumentos das coletas de dados}

\subsubsection{Questionário sociodemográfico e clínico}

Os indivíduos aptos para a pesquisa responderam um questionário semiestruturado para coleta de informações sociodemográficas (idade, sexo, escolaridade) e clínicas (estado clínico geral, lista de medicamentos em uso e autorrelato de patologias), considerando como condições clínicas: cardiopatias, hipertensão arterial sistêmica, doenças cerebrovasculares, diabetes mellitus, câncer, doenças neurodegenerativas e doenças do aparelho respiratório.

\subsection{Questionário de Matutinidade e Vespertinidade de Horne \& Ostberg (QMV-HO).}

Para verificar o cronotipo das crianças (preferência temporal para realizar atividades diárias), aplicou-se o Questionário de Matutinidade e Vespertinidade de Horne \& Ostberg (Horne; O’ Stberg, 1976; Benedito-Silva et al., 1990). Este instrumento consta de 19 perguntas com respostas de múltipla escolha que consideram as preferências de horários para os hábitos do sono e para o melhor desempenho das atividades realizadas corriqueiramente durante o dia, tais como alimentação, atividade física e trabalho.

\subsubsection{Escala de Sonolência de Epworth (ESE)}

Utilizou-se a Escala de Sonolência de Epworth, desenvolvida pelo Dr. Murray Johns (1991), a qual é uma ferramenta utilizada com frequência para aferir o grau de sonolência diurna. $O$ teste foi aplicado com cada criança no período da manhã em uma sala disponibilizada pela escola. Os entrevistados foram questionados sobre a percepção de sua tendência de adormecer ou dormir em oito situações diferentes em uma escala que varia de 0 (nunca dormiria) para 3 (alto risco de dormitar). O resultado de até 9 pontos indica uma condição considerada normal (Johns, 1991).

\subsubsection{Inventário de Ansiedade Traço-Estado (IDATE)}

O Inventário de Ansiedade Traço-Estado - IDATE (BIAGGIO; NATALÍCIO; SPIELBERGER, 1977) avalia duas dimensões de ansiedade, o estado transitório e o traço relativamente estável da personalidade do indivíduo. Cada dimensão consiste em vinte afirmações e, para cada afirmação, a criança assinalava uma das quatro alternativas, indicando como se sentia: (1) absolutamente não, (2) um pouco, (3) bastante e (4) muitíssimo (na escala IDATE-Estado); (1) quase nunca, (2) às vezes, (3) frequentemente e (4) quase sempre (na escala IDATE-Traço). O inventário é composto por afirmações positivas (benéficas) e negativas (maléficas), onde a pontuação de cada afirmação positiva tem o peso invertido. No IDATE Estado há 10 afirmações positivas $(1,2,5,8,10,11,15,16,19$ e 20) e 10 negativas $(3,4,6,7,9,12,13,14,17$ e 18); por outro lado, o IDATE Traço contém 7 afirmações positivas $(1,6,7,10,13,16$ e 19) e 13 negativas $(2,3,4,5,8,9,11,12,14,15,17,18$ e 
20). O escore total é caracterizado por grau leve de ansiedade (20-34 pontos), grau moderado de ansiedade (35-49), grau elevado de ansiedade (50-64) e grau muito elevado de ansiedade (65-80) (Andrade et al., 2001; Fioravanti et al., 2006).

O teste foi realizado com cada criança no período da manhã em uma sala privada. Para aqueles que sabiam ler (embora a idade e também o ano escolar das crianças, alguns não sabiam ler ou compreender corretamente), o pesquisador solicitou aos participantes que lessem as perguntas presentes no questionário com atenção e assinalassem um número de 1 a 4 que mais correspondesse a como eles estavam se sentindo no momento (IDATE-Estado) e como usualmente se sentiam no dia a dia (IDATE-Traço). Quanto maior o valor da escala, maior a severidade dos sintomas de ansiedade. Aos participantes que não sabiam ler ou tinham dificuldade de leitura, o pesquisador leu e explicou detalhadamente cada item do questionário.

\subsection{Análise de dados}

A análise descritiva dos dados foi realizada por meio de frequências absolutas e relativas para as variáveis categóricas; e das medidas de tendência central (média e desvio padrão ou mediana, mínimo e máximo) para as variáveis numéricas, como também o percentual das médias. Os resultados estão dispostos sob a forma de gráficos e tabelas.

Analisou-se a normalidade da distribuição dos dados pelo teste Kolmogorov-Smirnov. Para os dados paramétricos, utilizou-se o teste $\mathrm{t}$ de Student para comparação entre dois grupos e o teste ANOVA para comparação entre mais de dois grupos (com post-hoc de Tukey). Para os dados não paramétricos, aplicou o teste de Wilcoxon para comparação. Utilizou-se o teste de Spearman para realizar as correlações. Foi considerado o nível de significância $p<0,05$. As análises estatísticas foram realizadas através do programa GraphPad Prism (versão 8.2).

\section{Resultados}

\subsection{Perfil sociodemográfico e clínico dos participantes do estudo}

Ao aplicar-se os critérios de elegibilidade, obteve-se um total de 88 participantes de ambos os sexos, sendo 55,7\% do sexo masculino (n=56) e 44,3\% do sexo feminino ( $n=32$ ). Algumas crianças não participaram dos períodos inicial ou final da pesquisa devido à ausência no dia da coleta, assim como pela impossibilidade de se ausentar das aulas para a realização dos testes.

Em relação à idade, 22,2\% apresentavam 9 anos de idade ( $\mathrm{n}=19), 50,6 \%$ apresentavam 10 anos $(\mathrm{n}=44)$ e 27,2\% apresentavam 11 anos $(n=25)$. Ainda referente ao total de participantes, 20,5\% relataram apresentar algum problema de saúde; 2,3\% relataram apresentar alguma incapacidade física ou motora; $17 \%$ teriam alguma restrição alimentar; $3,4 \%$ faziam uso de medicamentos; e 100\% dos participantes residiam em bairros ou zonas rurais da cidade de Vitória de Santo Antão - PE.

\subsection{Qualidade do sono dos escolares}

Para avaliar a qualidade do sono dos escolares, levou-se em consideração o cronotipo e o grau de sonolência. Assim, 70 alunos participaram da análise do cronotipo, no qual obteve-se que, $80 \%$ dos participantes foram classificados como intermediários $(n=56), 10 \%$ como matutinos $(n=7)$ e $10 \%$ como vespertinos $(n=7)$. Não houve diferença estatisticamente significante do cronotipo entre os sexos dos escolares.

A avaliação do grau de sonolência contou com a participação de 69 escolares no período 1, 23\% dos escolares apresentaram um grau de sonolência normal; 38\% sonolência moderada; 38\% sonolência anormal, e 1\% apresentou grau de sonolência ruim. No período 2, repetiu-se o teste com os mesmos alunos $(\mathrm{n}=58)$, apesar da desistência de boa parte deles. Assim, 33\% apresentaram sonolência normal; 9\% sonolência moderada; 59\% sonolência anormal, e 0\% apresentaram sonolência ruim. Observa-se assim, que no período 2 houve uma diminuição da prevalência de escolares com sonolência 
classificada como moderada e um aumento no número de escolares com grau de sonolência anormal (Figura 1). Assim, vale ressaltar que

Figura 1 - Análise do grau de sonolência em escolares com idade entre 9 e 11 anos.

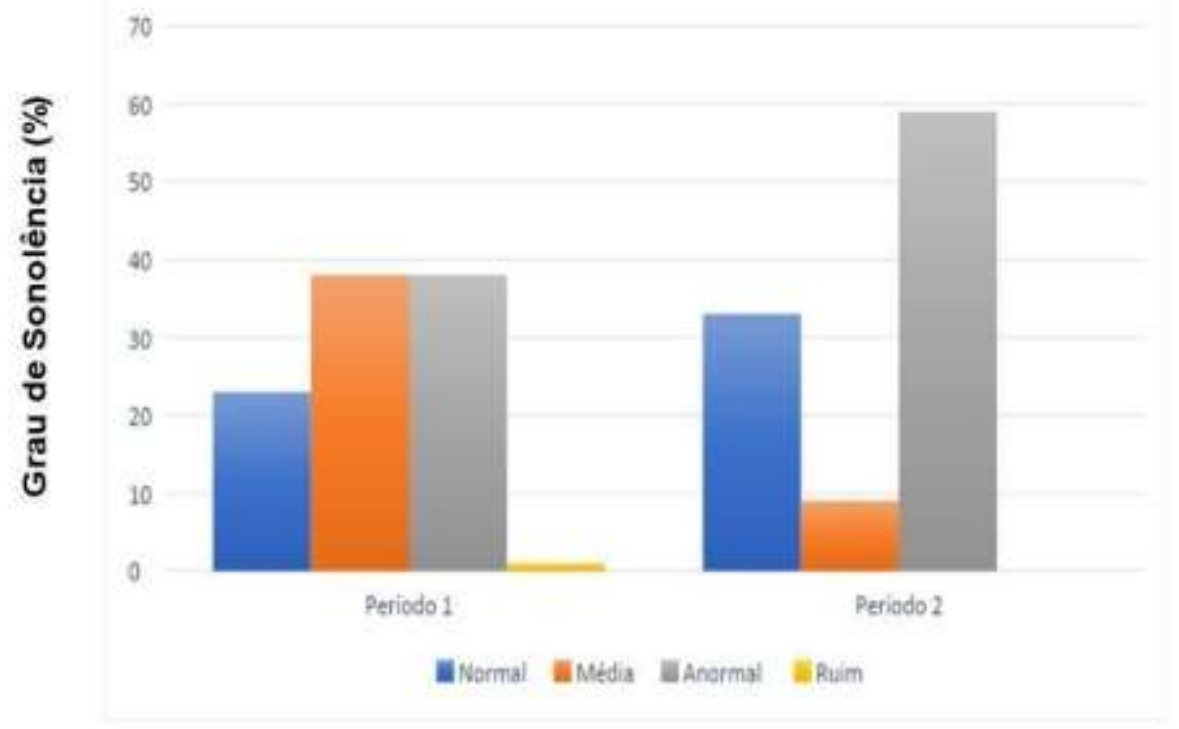

Realizado no período 1(n=69) e período 2 (n=58). Dados expressos em percentual das médias. Fonte: Autores (2021).

Comparando-se o grau de sonolência de acordo com a idade e sexo dos escolares, houve uma tendência à diminuição da diferença entre os valores nos períodos 1 e 2 para as idades de 9 (P1: 0,0;1,5; 3,0 (n=12) e P2: 0,0;0,0; 2,0 (n=11); $\mathrm{p}=0,052)$. Já em relação ao sexo, não houve diferença entre os períodos 1 e 2 (p>0,05).

\subsection{Níveis de ansiedade dos participantes avaliados}

A amostra contou com 63 estudantes que responderam o Inventário de Ansiedade Traço-Estado no período 1. No período 2 repetiu-se o teste com os mesmos alunos do período anterior $(n=57)$, contudo houve desistência de boa parte deles. Em termos de estado de ansiedade, evidenciou-se que no início do semestre letivo (P1), dos 63 participantes, 10 apresentaram ansiedade leve, 13 apresentaram ansiedade moderada, 4 apresentaram ansiedade elevada, nenhum dos participantes apresentou ansiedade muito elevada no IDATE-Estado (Figura 2), os demais participantes não apresentaram estado de ansiedade. No P2, 3 apresentaram ansiedade leve, 20 apresentaram ansiedade moderada, 4 apresentaram ansiedade elevada, nenhum dos participantes apresentou ansiedade muito elevada no IDATE-Estado (Figura 2), os demais participantes não apresentaram estado de ansiedade. Assim pode-se observar que houve uma diminuição na prevalência de ansiedade leve e um aumento na ansiedade moderada do IDATE-Estado.

No IDATE-Traço, ainda em P1, 7 crianças apresentaram ansiedade leve, 19 apresentaram ansiedade moderada, 6 apresentaram ansiedade elevada e nenhum dos participantes apresentaram ansiedade muito elevada (Figura 3), os demais 
Research, Society and Development, v. 10, n. 8, e17210817148, 2021

(CC BY 4.0) | ISSN 2525-3409 | DOI: http://dx.doi.org/10.33448/rsd-v10i8.17148

participantes não apresentaram nenhum dos traços para ansiedade. Não houve diferenças no IDATE-Traço entre os períodos 1 e $2(p>0,05)$.

Figura 2 - Avaliação do estado de ansiedade em escolares com idade entre 9 e 11 anos.

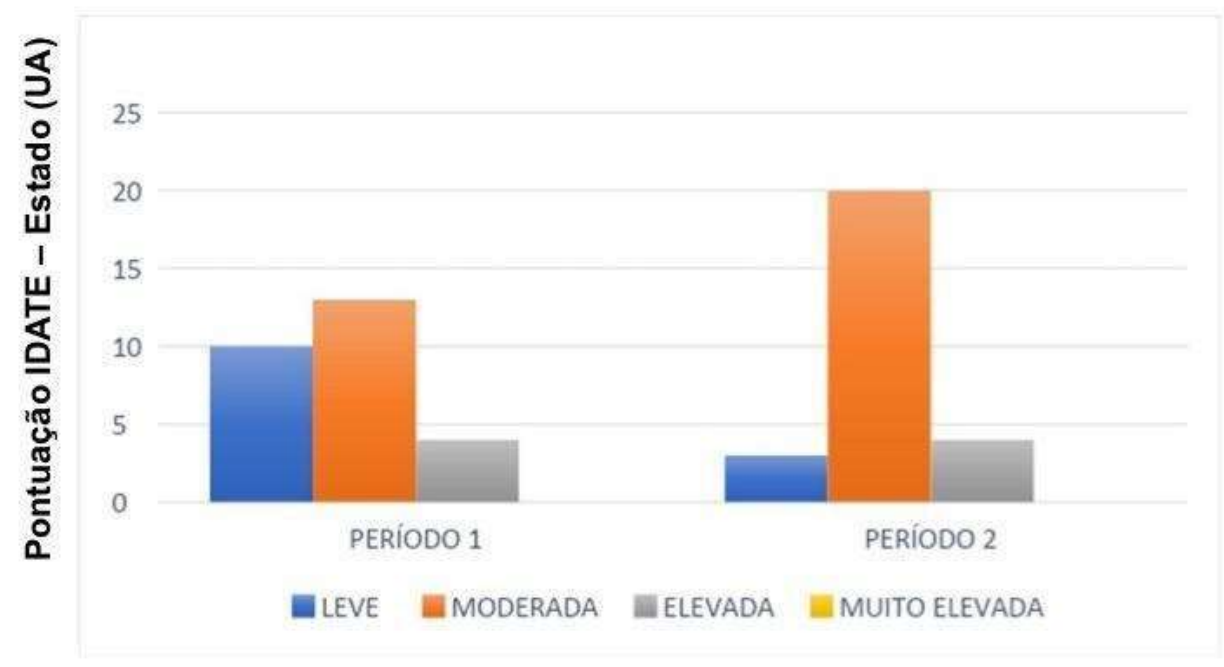

Realizado no período $1(\mathrm{n}=63)$ e no período $2(\mathrm{n}=57)$. Dados expressos em percentual das médias. IDATE= Inventário de Ansiedade Traço e Estado. Fonte: Autores (2021).

Figura 3 - Avaliação do traço de ansiedade em escolares com idade entre 9 a 11 anos.

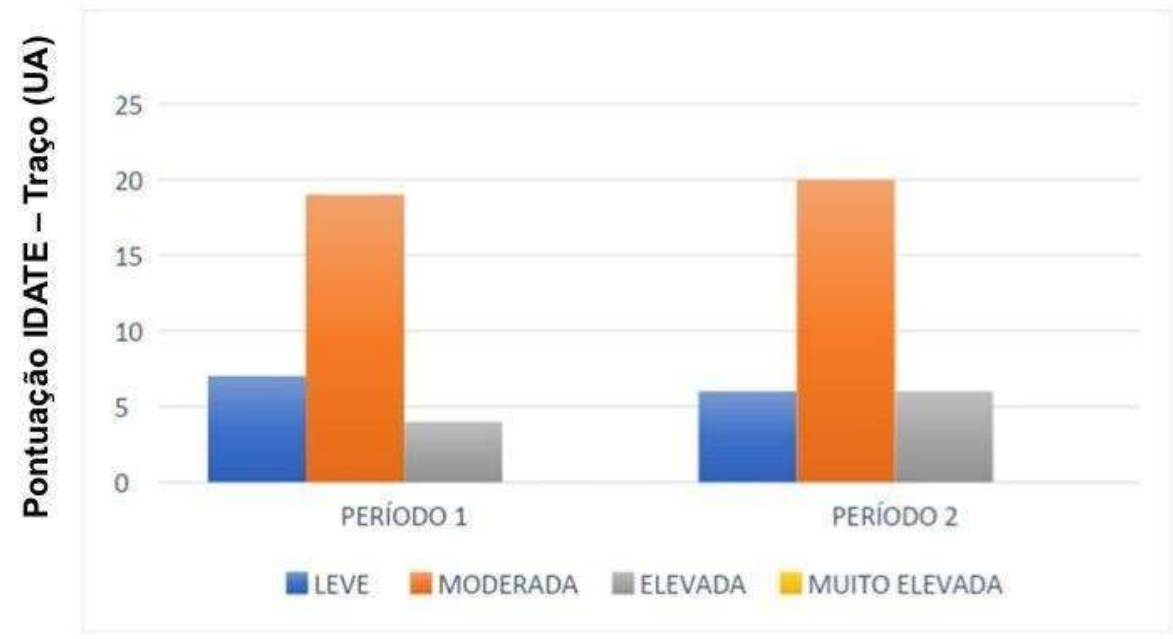

Realizado no período $1(\mathrm{n}=63)$ e no período 2 ( $\mathrm{n}=57)$. Dados expressos em percentual das médias. IDATE= Inventário de Ansiedade Traço e Estado. Fonte: Autores (2021). 
Não foi verificada diferença estatística nos níveis de ansiedade traço e estado ao comparar os dados totais entre o início e final do semestre escolar ( $>>0,05)$ (Tabela 1). Ao analisar os subcomponentes de cada escala, também não houve diferença quanto aos aspectos negativos ou positivos entre os dois momentos de avaliação ( $p>0,05$ ). Por fim, não foi observada diferença ao considerar a idade e o sexo das crianças entre o início e final do semestre escolar (p>0,05) (Tabelas 2 e 3).

Tabela 1 - Comparação dos níveis de ansiedade em escolares com idade entre 9 a 11 anos entre o início e final do semestre escolar.

\begin{tabular}{|c|c|c|}
\hline & $\begin{array}{c}\text { Período } 1 \\
\text { mínimo; mediana; máximo }(\mathrm{n}=63)\end{array}$ & $\begin{array}{c}\text { Período } 2 \\
\text { mínimo; mediana; máximo }(\mathrm{n}=57)\end{array}$ \\
\hline IDATE $-\mathrm{E}(\text { Total })^{\mathrm{a}}$ & 23,$0 ; 38,0 ; 53,0$ & 23,$0 ; 38,0 ; 59,0$ \\
\hline IDATE $-\mathrm{E}(+)^{\mathrm{a}}$ & 10,$0 ; 19,0 ; 33,00$ & 12,$0 ; 21,0 ; 38,0$ \\
\hline IDATE - E $(-)^{a}$ & 10,$0 ; 15,0 ; 40,00$ & 10,$0 ; 17,0 ; 31,0$ \\
\hline IDATE $-\mathrm{T}\left(\right.$ Total) ${ }^{\mathrm{b}}$ & 21,$0 ; 39,0 ; 55,00$ & 22,$0 ; 41,0 ; 56,0$ \\
\hline IDATE $-\mathrm{T}(+)^{\mathrm{a}}$ & 7,$0 ; 12,0 ; 23,00$ & 7,$0 ; 14,0 ; 29,0$ \\
\hline IDATE $-\mathrm{T}(-)^{\mathrm{b}}$ & 13,$0 ; 26,0 ; 42,00$ & 9,$0 ; 27,0 ; 46,0$ \\
\hline
\end{tabular}

Resultados expressos de acordo com as análises realizadas através do teste de Wilcoxon $\left({ }^{a}\right)$ e teste $t\left({ }^{b}\right)$, $\mathrm{p}>0,05$. IDATE $-\mathrm{E}(\mathrm{TOTAL})=$ Valores dos escores totais do IDATE - Estado; IDATE $-\mathrm{E}(+)=$ Valores dos escores das afirmações positivas do IDATE - Estado; IDATE - E (-) = Valores dos escores das afirmações negativas do IDATE - Estado; IDATE $-\mathrm{T}($ TOTAL $)=$ Valores dos escores totais do IDATE - Traço; IDATE $-\mathrm{T}(+)=$ Valores dos escores das afirmações positivas do IDATE - Traço; IDATE $-\mathrm{T}(-)=$ Valores dos escores das afirmações negativas do IDATE - Traço. Fonte: Autores (2021).

Tabela 2 - Comparação do estado de ansiedade em escolares entre o início e final do semestre escolar de acordo com a idade e sexo.

\begin{tabular}{lcc}
\hline Idades/sexo & \multicolumn{2}{c}{ Período 1} \\
& mínimo; mediana; máximo (n) & Período 2 \\
\hline 9 anos & 23,$0 ; 34,0 ; 52,0(15)$ & 24,$0 ; 35,5 ; 46,0(10)$ \\
10 anos & 23,$0 ; 42,0 ; 53,0(29)$ & 23,$0 ; 39,0 ; 59,0(29)$ \\
11 anos & 28,$0 ; 37,5 ; 52,0(18)$ & 30,$0 ; 38,0 ; 51,0(17)$ \\
Feminino & 27,$0 ; 40,0 ; 53,0(33)$ & 23,$0 ; 37,0 ; 59,0(31)$ \\
Masculino & 23,$0 ; 37,0 ; 52,0(30)$ & 27,$0 ; 38,0 ; 51,0(26)$ \\
\hline
\end{tabular}

Resultados expressos de acordo com as análises realizadas através do teste ANOVA e post hoc de Tukey, p>0,05; n= amostra. Fonte: Autores (2021).

Tabela 3 - Comparação do traço de ansiedade em escolares entre o início e final do semestre escolar de acordo com a idade e sexo.

\begin{tabular}{lcc}
\hline Idades/sexo & Período 1 & Período 2 \\
& mínimo; mediana: máximo (n) & mínimo; mediana: máximo (n) \\
\hline 9 anos & 27,$0 ; 38,0 ; 51,0(15)$ & 23,$0 ; 39,5,51,0(10)$ \\
10 anos & 21,$0 ; 40,0,53,0(29)$ & 22,$0 ; 41,0 ; 56,0(29)$ \\
11 anos & 26,$0 ; 38,5 ; 55,0(18)$ & 32,$0 ; 43,0 ; 55,0(17)$ \\
Feminino & 28,$0 ; 40,0 ; 53,0(33)$ & 23,$0 ; 41,0 ; 56,0(31)$ \\
Masculino & 21,$0 ; 37,0 ; 55,0(30)$ & 22,$0 ; 42,0 ; 55,0(26)$ \\
\hline
\end{tabular}

Resultados expressos de acordo com as análises realizadas através do teste ANOVA e post hoc de Tukey, p>0,05; n= amostra. Fonte: Autores (2021). 


\subsection{Correlação entre sonolência e níveis de ansiedade dos escolares}

Os dados mostram uma correlação insuficiente entre sonolência e o IDATE-Estado no período $1(\mathrm{r}=0,16 ; \mathrm{p}=0,21)$, bem como no período $2(\mathrm{r}=0,34 ; \mathrm{p}=0,01)$. Essa correlação baixa também foi observada entre a sonolência e os aspectos IDATE-Traço no período $1(\mathrm{r}=0,15 ; \mathrm{p}=0,24)$, porém no período 2 , a correlação foi moderada $(\mathrm{r}=0,48$; $\mathrm{p}<0,001)$. Nos aspectos positivos e negativos a correlação da sonolência IDATE-Traço e Estado apresentaram-se com correlação de baixa a moderada (Tabela 4).

Tabela 4 - Correlação da sonolência e os níveis de ansiedade dos escolares entre o início e final do semestre escolar.

\begin{tabular}{lcc}
\hline $\begin{array}{c}\text { Subcomponentes } \\
\text { IDATE }\end{array}$ & $\begin{array}{c}\text { Sonolência } \\
\text { Período 1 }\end{array}$ & $\begin{array}{c}\text { Sonolência } \\
\text { Período 2 }\end{array}$ \\
\hline $\begin{array}{l}\text { IDATE- E (Total) P1 } \\
\text { IDATE- E (Total) P2 }\end{array}$ & $(\mathrm{r}=0,16 ; \mathrm{p}=0,21)$ & \\
IDATE- T (Total) P1 & $(\mathrm{r}=0,15 ; \mathrm{p}=0,24)$ & $(\mathrm{r}=0,34 ; \mathrm{p}=0,01)$ \\
IDATE- T (Total) P2 & & $(\mathrm{r}=0,48 ; \mathrm{p}<0,001)$ \\
IDATE- E (+) P1 & $(\mathrm{r}=0,020 ; \mathrm{p}=0,88)$ & \\
IDATE- E (+) P2 & & $(\mathrm{r}=0,004 ; \mathrm{p}=0,97)$ \\
IDATE- E (-) P1 & $(\mathrm{r}=0,18 ; \mathrm{p}=0,18)$ & \\
IDATE- E (-) P2 & & $(\mathrm{r}=0,47 ; \mathrm{p}<0,01)$ \\
IDATE- T (+) P1 & $(\mathrm{r}=0,32 ; \mathrm{p}=0,80)$ & \\
IDATE- T (+) P2 & & $(\mathrm{r}=0,007 ; \mathrm{p}=0,96)$ \\
IDATE- T (-) P1 & $(\mathrm{r}=0,14 ; \mathrm{p}=0,28)$ & \\
IDATE- T (-) P2 & & $(\mathrm{r}=0,49 ; \mathrm{p}<0,01)$ \\
\hline
\end{tabular}

Resultados expressos de acordo com as análises realizadas através do teste Spearman. $\mathrm{p}<0,05$. IDATE $-\mathrm{E}(\mathrm{TOTAL}) \mathrm{P} 1=$ Valores dos escores totais do IDATE - Estado no período 1; IDATE - E (+) P1 = Valores dos escores das afirmações positivas do IDATE - Estado no período 1; IDATE - E (-) P1= Valores dos escores das afirmações negativas do IDATE - Estado no período 1; IDATE - T (TOTAL) P1 = Valores dos escores totais do IDATE - Traço no período 1; IDATE $-\mathrm{T}(+) \mathrm{P} 1=$ Valores dos escores das afirmações positivas do IDATE - Traço no período 1; IDATE - T (-) P1= Valores dos escores das afirmações negativas do IDATE - Traço no período 1. IDATE - E (TOTAL) P2 $=$ Valores dos escores totais do IDATE - Estado no período 2; IDATE $-\mathrm{E}$ (+) P2 = Valores dos escores das afirmações positivas do IDATE - Estado no período 2; IDATE $-\mathrm{E}(-) \mathrm{P} 2=$ Valores dos escores das afirmações negativas do IDATE - Estado no período 2; IDATE - T (TOTAL) P2= Valores dos escores totais do IDATE - Traço no período 2; IDATE $-\mathrm{T}(+) \mathrm{P} 2=$ Valores dos escores das afirmações positivas do IDATE - Traço no período 2; IDATE - T (-) P2 = Valores dos escores das afirmações negativas do IDATE - Traço no período. $\mathrm{r}=$ força da correlação. $\mathrm{p}=$ nível de significância. $\mathrm{x}=$ Não aplicável. Fonte: Autores (2021).

\section{Discussão}

O presente estudo mostra que houve predominância de crianças com o cronotipo intermediário e aumento da prevalência do grau de anormalidade na sonolência no final do semestre escolar. Essas mudanças no grau de sonolência apresentam relação moderada com estado e traços de ansiedade nos escolares avaliados.

Corroborando com os nossos achados, um estudo verificou que o número de crianças com o cronotipo intermediário foi maior que o número de crianças com os cronotipos matutino e vespertino (Eid et al., 2020). O fato de grande maioria das crianças do presente estudo apresentarem um cronotipo intermediário pode vir a auxiliar no desempenho das suas atividades escolares, visto que indivíduos com indiferença no seu cronotipo tem maior facilidade para realizar suas atividades em ambos os turnos do dia. Pessoas com cronotipo intermediário são aquelas que conseguem manter o ritmo de desempenho do organismo tanto pela manhã quanto a tarde (com pico de melatonina por volta das $3 \mathrm{~h}$ da manhã) e dormem geralmente entre meia noite e $8 \mathrm{~h}$ da manhã, correspondendo a metade da população mundial (Taylor \& Hasler, 2018). 
Uma boa qualidade do sono é essencial para o bom desempenho escolar (O’Brien et al., 2019). No presente trabalho, o grau de sonolência diurna moderada no início do período escolar é preocupante visto que a faixa etária dos participantes do estudo são crianças de 9 a 11 anos de idade. No final do semestre, os dados mostram que há um aumento da anormalidade no grau de sonolência diurna. Estudos com crianças com faixa etária de 6 a 12 anos mostraram que o grau de sonolência diurna excessiva pode ocasionar interrupções na cognição (Jackson et al., 2011), o que pode prejudicar o desempenho das crianças nas atividades escolares.

A anormalidade da sonolência diurna, pode ser causada por fatores como o local onde a criança dorme e o ambiente escolar, assim como o contexto socioeconômico em que a criança vive. Um estudo longitudinal mostrou que ambientes com iluminação inadequada durante as fases do dia podem interferir na produção e secreção de melatonina fazendo com que essa anormalidade do sono se torne cada vez mais frequente entre as pessoas (Touitou et al., 2017). Um dos fatores que pode afetar o horário do sono entre as crianças é o uso inadequado e tardio de equipamentos eletrônicos (Macchitella et al., 2020).

Aspectos nutricionais e a rotina escolar também podem estar relacionadas à anormalidade da sonolência diurna dos escolares. Um estudo aponta que o déficit de alguns micronutrientes pode causar uma desregulação metabólica e fisiológica (prejudicando o funcionamento de órgãos vitais e contribuindo para o desenvolvimento de doenças, como obesidade e diabetes), resultando em impacto negativo sobre a qualidade do sono dos indivíduos (Ji et al., 2018). Considerando que os escolares que participaram do estudo são predominantemente pertencentes a um status socioeconômico baixo e muitas vezes não têm acesso a uma alimentação equilibrada de vitaminas e minerais, esse fator pode estar relacionado à anormalidade da sonolência evidenciada.

Outro ponto importante a ser discutido é a relação entre sono e atividade física visto que ambos podem impactar no rendimento um do outro. Um estudo que analisou a relação entre obesidade, sono e atividade física evidenciou que quanto menos a criança se exercita maiores são as chances de desenvolver obesidade, e quanto mais obesa a criança for, maior dificuldade para dormir ela terá (Ji et al., 2018). Tendo em vista que o uso excessivo e precoce de equipamentos eletrônicos por crianças pode reduzir o interesse na prática de atividade física regular, faz-se necessária a inserção de aulas de Educação Física para este público (Macchitella et al., 2020). Em especial na escola onde o presente estudo foi realizado, pois não apresenta profissionais de Educação Física para a realização de aulas práticas. Podendo também ser sugerido a realização de futuros estudos para realizar a comparação das variáveis de ansiedade e sonolência entre crianças submetidas à Educação Física e sem Educação Física.

Um estudo na literatura analisou o horário de início das aulas, o desempenho escolar e a duração do sono de crianças a partir de alguns questionários de análise subjetiva e objetiva do sono, dentre eles o Índice de Qualidade de Sono de Pittsburgh. Foi evidenciado um aumento da quantidade de horas de sono no decorrer do ano letivo e uma melhora no desempenho escolar no fim do ano letivo (Alfonsi et al., 2020). Levando em consideração que os dados do presente estudo também mostram que o grau de sonolência decaiu no final do semestre letivo, uma das hipóteses para esse agravo pode ser o estresse gerado pela rotina escolar durante o semestre letivo. Algumas mudanças comportamentais dos alunos podem ser refletidas na qualidade do sono que, por sua vez, poderia prejudicar o rendimento escolar das crianças.

Os resultados da presente pesquisa apontam para o aumento do estado de ansiedade no final do semestre escolar, o que pode ter sido ocasionado por alguns estímulos como o estresse causado pelas tarefas escolares. Um estudo com crianças, na faixa etária entre 8 e 12 anos, que também utilizou o IDATE, evidenciou relação entre a ansiedade e o estresse (verificado pelo cortisol salivar) nas crianças (Queiroz et al., 2015). Tendo em vista que não se avaliou o estresse dos escolares, ressaltamos que uma hipótese para esse aumento do grau de ansiedade moderada pode estar relacionada à anormalidade na sonolência dos escolares durante o semestre letivo. 
Outro estudo analisou o sono e o status socioeconômico de crianças com a mesma faixa etária do presente estudo e revelou que crianças mais pobres apresentaram pior qualidade do sono em relação às crianças com o nível socioeconômico mais elevado (Philbrook et al., 2017). Similarmente, um estudo brasileiro relacionando o contexto social e a saúde mental infantil mostrou que os sintomas de ansiedade e depressão foram predominaram entre crianças com faixa etária entre 6 e 12 anos com vulnerabilidade socioeconômica (Ferriolli et al., 2017). Esta é uma questão preocupante, pois interfere negativamente no desenvolvimento social das crianças, podendo fazer com que as crianças se tornem adultos ansiosos.

Tendo em vista que os dados do presente estudo foram coletados em uma escola municipal localizada numa comunidade carente que atende crianças com o nível socioeconômico baixo, pode haver relação entre a questão socioeconômica, a qualidade do sono e os sintomas ansiosos. Fatores que envolve o ambiente de dormir, relacionados à higiene do sono, por exemplo: o contexto em que essas crianças estão inseridas, as interações familiares diferentes, muitas vezes com baixa condição econômica, em um ambiente desfavorecido de uma iluminação adequada e todo o meio que envolve seu ambiente de dormir, todo esse contexto pode interferir na qualidade do sono da criança (Fusz et al., 2019). Esse contexto, pode agravar a anormalidade do sono, o que pode ocasionar algumas consequências negativas como problemas emocionais, dificuldade de aprender e raciocinar, interferindo assim no seu rendimento escolar. Esses problemas na qualidade do sono também podem causar outros problemas mentais e físicos no decorrer da vida (Matthews \& Pantesco., 2016; Paavonen et al., 2010). Portanto, sugere-se para trabalhos futuros, a realização de análises mais específicas do sono, como polissonografia e actigrafia, com a finalidade de identificar alterações na arquitetura do sono e no ciclo sono-vigília, respectivamente. Além disso, faz-se necessário o estudo de outros aspectos emocionais, como o estresse e a depressão, para que se tenha um panorama mais completo do perfil emocional das crianças.

Apesar das evidências encontradas nesta pesquisa serem relevantes para a sociedade e para a área da Educação Física, uma vez que mostram que as crianças estão apresentando problemas de sono e sintomas de ansiedade, servindo de alerta para as autoridades das escolas e para os familiares responsáveis, este estudo apresenta algumas limitações, como o baixo quantitativo de alunos participantes da pesquisa, utilização de instrumentos de avaliação subjetiva e utilização de métodos não invasivos, bem como o curto período de avaliação ( 1 semestre).

Sabe-se que a prática de atividades físicas no âmbito escolar pode favorecer a integração social da criança, desenvolvimento físico e mental, bem como pode fazer com que a criança passe a se sentir mais fadigado durante o dia, fazendo com que ela tenha uma melhora no seu sono durante a noite (Oliveira., 2018; Silva., 2020; Sauders.,2016; Reid., 2010). Assim, o conhecimento sobre os aspectos da ritmicidade biológica torna-se algo importante para o conhecimento de todos, principalmente o ciclo sono-vigília, pois mostra que cada criança tem sua individualidade. No ambiente escolar é necessário entender que a qualidade e o tempo do sono podem interferir no desempenho dos escolares. Além do mais, problemas no sono podem se tornar barreiras na aprendizagem desses escolares no futuro.

\section{Conclusão}

Os resultados revelam que os alunos avaliados apresentam anormalidade na sonolência diurna ao final do semestre letivo e tais alterações podem favorecer o desenvolvimento de traços e estados de ansiedade. Todavia, no início do semestre letivo as crianças apresentaram menos sintomas de ansiedade comparado ao final do semestre. Os sintomas ansiosos podem interferir na aprendizagem e socialização dos escolares, prejudicando o seu desempenho físico e mental.

\section{Referências}

Alfonsi, V., Palmizio, R., Rubino, A., Scarpelli, S., Gorgoni, M., D'Atri, A., Pazzaglia, M., Ferrara, M., Giuliano, S., \& De Gennaro, L. (2020). The Association Between School Start Time and Sleep Duration, Sustained Attention, and Academic Performance. Nature and science of sleep, $12,1161-1172$. 
AlmondeS, K. M., \& Araújo, J. F. (2003). Padrão do ciclo sono- vigília e sua relação com a ansiedade em estudantes universitários. Estudos de Psicologia, $8(1), 37-43$

Andrade, L., Gorenstein, C., Vieira Filho, A. H., Tung, T.C., \& Artes, R. (2001). Psychometric properties of the Portuguese version of the State-Trait Anxiety Inventory applied to college students: factor analysis and relation to the Beck Depression Inventory. Brazilian Journal of Medical and Biological Research, 34(3), 367-374.

Benedito-Silva, A. A., Menna-Barreto, L., Marques, N., \& Tenreiro, S. (1990). A self-assessment questionnaire for the determination of morningnesseveningness types in Brazil. Progress in Clinical and Biological Research, 341B, 89-98.

Bertolazi, A. N., Fagondes, S. C., Hoff, L. S., Dartora, E. G., Miozzo, I. C., de Barba, M. E., \& Barreto, S. S. (2011). Validation of the Brazilian Portuguese version of the Pittsburgh sleep quality index. Sleep Medicine, 12(1), 70-75.

Bueno, C., \& Wey, D. (2018). Gênese e ontogênese do ritmo de sono/vigília em humanos. Revista da Biologia, 9(3), 62-67.

Callear, A., Harvey, S. T., Bimler, D., \& Catto, N. (2018). Profiling children's emotion regulation behaviours. British Journal of Developmental Psychology, $36(4), 540-556$

Campos, T. F., Pinheiro, C. D., Diógenes, F. P., Barroso, M. T., \& Dantas, A. A. (2011). Chronotype and implications for its use in physical therapy in patients with stroke. Fisioterapia e Pesquisa, 18 (3), 258-263.

Eid, B., Bou, S. M., Melki, I., Torbey, P., Najem J., Saber, M., El, O. N., \& Rabbaa, K. L. (2020). Evaluation of Chronotype Among Children and Associations With BMI, Sleep, Anxiety, and Depression. Frontiers in Neurology, 11, 416.

Fallone, G.; Owens, J. A.; \& Deane, J. (2002). Sleepiness in children and adolescents: clinical implications. Sleep medicine reviews, 6(4), 287-306.

Ferriolli, S. H. T., Marturano, E. M., \& Puntel, L. P. (2007). Contexto familiar e problemas de saúde mental infantil no Programa Saúde da Família. Revista de Saúde Pública, 41, 251-259.

Fioravanti, A. C. M., Santos, L. F., Maissonette, S., Cruz, A. P. M., \& Landeira-Fernandez, J. (2006). Avaliação da estrutura fatorial da Escala de AnsiedadeTraço do IDATE. Avaliação Psicológica, 5(2), 217-224.

Fusz, K., Ritecz, B., Balogh, B., Takács, K., Somlai, E., Raposa, L. B., \& Oláh, A. (2019). Sleep habits among preschool-and schoolchildren. Ideggyogyaszati szemle, 72(7-8), 264-272.

Gondim, S. M. G., Pereira, C.R., Hirschle, A.L.T., Palma, E. M. S., Alberton, G. D., Paranhos, J., Santana, V., Ribeiro, W. R. B. (2015). Evidências de Validação de uma Medida de Características Pessoais de Regulação das Emoções. Psicologia: Reflexão e Crítica, 28(4), 659-667.

Gustafsson, M. L., Laaksonen, C., Aromaa, M., Asanti, R., Heinonen, O. J., Koski, P., Koivusilta, L., Löyttyniemi, E., Suominen, S., \& Salanterä, S. (2016). Association between amount of sleep, daytime sleepiness and health-related quality of life in schoolchildren. Journal of advanced nursing, 72(6), $1263-1272$.

Halal, C. S., \& Nunes, M. L. (2014). Education in children's sleep hygiene: which approaches are effective? A systematic review. Jornal de pediatria, 90(5), $449-456$.

Heins, E., Seitz, C., Schüz, J., Toschke, A. M., Harth, K., Letzel, S., \& Böhler E. (2007). Bedtime, television and computer habits of primary school children in Germany. Gesundheitswesen, 69(3),151-7.

Horne, J. A \& Stberg, O. (1976). A self-assessement questionnaire to determine morningness-eveningness in human circadian rhythms. Int. J. Chronobiol, $4(2), 97-110$.

Iwadare, Y., Kamei, Y., Usami, M., Ushijima, H., Tanaka, T., Watanabe, K., Kodaira, M., \& Saito, K. (2015). Behavioral symptoms and sleep problems in children with anxiety disorder. Pediatrics International, 57 (4), 690-3.

Jackson, M. L., Croft, R. J., Kennedy, G. A., Owens, K., \& Howard, M. E. (2013). Cognitive components of simulated driving performance: Sleep loss effects and predictors. Accid Anal Prev, 50, 438-44.

Ji, Y., Li, M., Zhang, X., Peng, Y., \& Wen, F. (2018). Poor Sleep Quality Is the Risk Factor for Central Serous Chorioretinopathy. Journal of ophthalmology, $2018,9450297$.

Johns, M. W.(1991). A new method for measuring daytime sleepiness: The Epworth Sleepiness Scale. Sleep, 14, 540-545.

Kim, J. H., \& Duffy, J. F. (2018). Circadian rhythm sleep-wake disorders in older adults. Sleep Medicine Clinics, 13(1), 39-50.

Kirwan, M., Pickett, S. M., \& Jarrett, N. L. (2017). Emotion regulation as a moderator between anxiety symptoms and insomnia symptom severity. Psychiatry Research, 254, 40-47.

Oliveira, W. P. P. D. (2018). Educação Física Escolar: Desenvolvimento Motor, Socialização E Prevenção.

Macchitella, L., Marinelli, C. V., Signore, F., Ciavolino, E., \& Angelelli, P. (2020). Sleepiness, Neuropsychological Skills, and Scholastic Learning in Children. Brain Sciences, 10(8), 529.

Martins T, Gomes CRG. (2010) Cronobiologia dos indivíduos em situação de trabalho. Revista Saúde e Pesquisa, 3 (3): 309-314.

Matricciani, L., Paquet, C., Galland, B., Short, M., \& Olds, T. (2019). Children's sleep and health: A meta-review. Sleep medicine reviews, 46, 136-150. https://doi.org/10.1016/j.smrv.2019.04.011. 
Matthews, K. A., \& Pantesco, E. J. (2016). Sleep characteristics and cardiovascular risk in children and adolescents: an enumerative review. Sleep medicine, $18,36-49$.

Menna-Barreto, Luiz, \& Marques, Nelson. (2002). O tempo dentro da vida, além da vida dentro do tempo. Ciência e Cultura, 54 (2), $44-46$.

McMakin, D. L., \& Alfano, C. A. (2015). Sleep and anxiety in late childhood and early adolescence. Current Opinion in Psychiatry, $28(6), 483$.

Moreira, G. A., \& Pradella-Hallinan, M. (2017). Sleepiness in Children: An Update. Sleep medicine clinics, 12(3), 407-413. https://doi.org/10.1016/j.jsmc.2017.03.013

Moro, J., Santos, P., Giacomin, A., Cardoso M., \& Bolan, M. (2021). Associação entre problemas para dormir e condições orais em escolares. Rev. paul. Pediatr., 39, e2019342.

O'Brien, L. M., Ivanenko, A., Crabtree, V. M., Holbrook, C. R., Bruner, J. L., Klaus, C. J., \& Gozal D. (2003). Sleep disturbances in children with attention deficit hyperactivity disorder. Pediatric Research, 54(2), 237-243.

Owens, J., \& Mindell, J. A. (2011). Sleep in children and adolescents, an issue of pediatric clinics-E-Book. Elsevier Health Sciences.

Silva, R. C. F. D. (2020). Hábitos de sono e de atividade física e desportiva das crianças do $1^{\text {o }}$ ciclo do ensino básico de Évora (Master's thesis, Universidade de Évora).

Silva, F. G., Silva, C. R., Braga, L. B., \& Neto, A. S. (2013). Hábitos e problemas do sono dos dois aos dez anos: estudo populacional. Acta Pediatr Port, 44(5), 196-202.

Paavonen, E. J., Räikkönen, K., Pesonen, A. K., Lahti, J., Komsi, N., Heinonen, K., \& Porkka-Heiskanen, T. (2010). Sleep quality and cognitive performance in 8-year-old children. Sleep medicine, 11(4), 386-392.

Palmer, C. A., \& Alfano, C. A. (2017). Sleep and emotion regulation: An organizing, integrative review. Sleep medicine reviews, 31, 6-16. https://doi.org/10.1016/j.smrv.2015.12.006.

Pavlova, M. (2017). Circadian rhythm sleep-wake disorders. Continuum: Lifelong Learning in Neurology, 23(4), 1051-1063.

Queiroz, A. M., Carvalho, A. B., Censi LL, Cardoso CL, Leite-Panissi CR, da Silva RA, de Carvalho FK, Nelson-Filho P, da Silva LA. (2015). Stress and anxiety in children after the use of computerized dental anesthesia. Brazilian Dental Journal, 26 (3), 303-7.

Reid, K. J., Baron, K. G., Lu, B., Naylor, E., Wolfe, L., \& Zee, P. C. (2010). Aerobic exercise improves self-reported sleep and quality of life in older adults with insomnia. Sleep medicine, 11(9), 934-940.

Reyes, A. N., Molina, M. L., Jansen, K., De Lima Bach, S., Do Amaral, P. L., Spessato, B. C., \& Silva, R. (2019). Biological rhythm and emotional and behavioral problems among schoolchildren in Southern Brazil. Chronobiology international, 36(3), 353-359. https://doi.org/10.1080/07420528.2018.1545781.

Saunders, T. J., Gray, C. E., Poitras, V. J., Chaput, J. P., Janssen, I., Katzmarzyk, P. T., Olds, T., Connor Gorber, S., Kho, M. E., Sampson, M., Tremblay, M. S., \& Carson, V. (2016). Combinations of physical activity, sedentary behaviour and sleep: relationships with health indicators in school-aged children and youth. Applied physiology, nutrition, and metabolism = Physiologie appliquee, nutrition et metabolisme, 41(6 Suppl 3), S283-S293.

Taylor, B. J., \& Hasler, B. P. (2018). Chronotype and mental health: recent advances. Current Psychiatry Reports, 20(8): 1-10, 2018.

Touitou, Y., Reinberg, A., \& Touitou, D. (2017). Association between light at night, melatonin secretion, sleep deprivation, and the internal clock: Health impacts and mechanisms of circadian disruption. Life sciences, 173, 94-106.

Touitou, Y., Touitou, D., \& Reinberg, A. (2016). Disruption of adolescents' circadian clock: The vicious circle of media use, exposure to light at night, sleep loss and risk behaviors. Journal of Physiology, 110(4), 467-479.

Xiao, Q., Chaput, J. P., Olds, T., Fogelholm, M., Hu, G., Lambert, E. V., Maher, C., Maia, J., Onywera, V., Sarmiento, O. L., Standage, M., Tremblay, M. S., Tudor-Locke, C., Katzmarzyk, P. T., \& ISCOLE Research Group (2020). Sleep characteristics and health-related quality of life in 9- to 11-year-old children from 12 countries. Sleep health, 6(1), 4-14. https://doi.org/10.1016/j.sleh.2019.09.006. 